\title{
Performance Comparison of Queue Management Algorithms in LTE Networks using NS-3 Simulator
}

\author{
Muhammet ÇAKMAK, Zafer ALBAYRAK*, Cumhur TORUN
}

\begin{abstract}
One of the most important issues accepted by researchers in LTE cellular systems is to develop Queue Management Algorithms for RLC (Radio Link Control). The performance of queue-management algorithms depends on parameters such as latency, packet dropping, and bandwidth usage. Simulation software is used to evaluate the queue-management algorithms developed and to test their performance. In the literature, active queue management algorithms have been compared with wired and wireless networks. In contrast to prior works, in this study, we have analyzed active queue management algorithms using the LTE model in the NS-3 network simulator. When the data and the results obtained from the simulations have been evaluated, it is concluded that the RED algorithm using probabilistic methods and the threshold value is more successful than the other algorithms in LTE networks.
\end{abstract}

Keywords: active queue management; cellular networks; LTE; NS-3 simulation

\section{INTRODUCTION}

The cellular wireless industry has been witnessing tremendous growth over the last 10 years with four billion wireless devices subscribers worldwide [1]. While mobile communications, which began early in the 1980s, initially allowed analogue voice transmission, nowadays it has become digital. In mobile communication technologies, improvements in services offered in the air interface and users are called generations. LTE Technology, a fourthgeneration communications $(4 \mathrm{G})$ technology, is end-to-end IP based system.

The Technologies that provide LTE and its development include OFDM (Orthogonal FrequencyDivision Multiplexing), SC-FDM (Single-Carrier Frequency-Division Multiplexing), MIMO (MultipleInput and Multiple-Output), Turbo coding and dynamic link adaptation techniques [2]. MIMO methods are used in mobile communication to increase the overall data rates and reliability of the communication link. It has been observed that the spectral efficiency increases with the use of the MIMO technique developed in LTE Technology [3].

One of the biggest problems in communication systems is packet loss. If a packet disappears before reaching the destination, the resources used for transmission are wasted [4]. In packet-switched networks, packages arrive late to the desired destination or become blocked; then the congestion occurs as a result. Queue management algorithms have been developed to prevent bottlenecks. It is aimed to reduce the accumulation of packets in the queue by the algorithms before the bottleneck.

Testing of technologies and protocols developed for network systems on real systems is both costly and laborious in terms of both material and time. Network Simulation Software has been developed so that such situations can be tested. Computer simulation of the network environment and testing of how the network will work without physical installation of the network is called network simulation. There are many open network and commercial simulations software used in research and development of new algorithms.
In the simulation performed for the LTE architecture, the RED, Droptail, CoDeL and pFIFO queue management algorithms are evaluated according to performance parameters such as packet loss, packet loss rate and end-toend delay time for different user numbers.

The rest of the paper is organized as follows: in Section II, we will review LTE architecture, queue management algorithms and network simulators as given in the literature. Section III contains the background of the LTE network and queue management algorithms. Section IV contains the NS-3 simulation framework for queue management algorithms and finally, Section V concludes the paper.

\section{RELATED WORK}

The characteristics of the simulation programs used in the LTE cellular systems were examined and the queue management algorithms were tested in different network environments.

In the work of Reddy and his colleagues in 2008, the performance of the RED and Droptail queue management algorithms in the TCP network environment was compared with the NS-2 simulation software. In the simulation, the bottleneck was followed by queuing management algorithms on a topology-oriented router. The simulation results show that the RED algorithm has a higher efficiency than the Droptail [5].

Arash Dana and his colleagues compared the active and passive queue management algorithms in the study they conducted in 2010. In the study made for OpNET Modeler, Droptail for the passive queue management algorithm and the RED algorithm for the active queue management algorithm were selected and compared. As a result of the study, it is observed that the RED algorithm has a low packet drop rate and delay, and also increases bandwidth usage [6].

In [7], the RED algorithm developed for WIMAX wireless networks was tested in OpNET Modeler simulation software. It has been seen that the algorithm developed has yielded more successful results in high traffic. 
The study of Albayrak and Torun in 2017 examines the simulation software used for LTE cellular systems and concludes that the NS-3 simulation software is more successful than the others [8].

The smRED-i algorithm for cellular networks was developed by Kumar et al [9] in 2017. This algorithm aims to prevent packets from dropping and packet delay in different load situations in the RLC buffer in evolved Node $\mathrm{B}$ (eNodeB). By changing the i parameter in the algorithm according to different load conditions, bottlenecks in the network are prevented.

Sharma and Behara in their study in 2017 tested the RED algorithm they developed for TCP/IP networks in the NS-2 simulation software. When compared to the RED algorithm, they found that the algorithm provides lower latency and higher bandwidth utilization [10].

In 2018 Zarrini \& Ghasemi investigated the effect of buffer size on the QoS of M2M traffic in $4 \mathrm{G}$ cellular networks. Their simulation results show that for the coordinated traffic model increasing the buffer size threshold is not effective in decreasing the packet loss probability of user equipment [11].

Adesh \& Renuka proposed a new congestion feedback mechanism to reduce queue overflow and queue delay for cellular networks. For this purpose, the sender congestion window is adjusted for low, medium and high load conditions. Also, packets are divided into TCP and UDP. A low range has been set for TCP because it is not resistant to drops. High ranges are determined for UDP, which is resistant to packet drops. Thus, the traffic changes according to the package type [12].

Cakmak \& Albayrak in 2021 tested performance of active queue management algorithms in LTE network using NS-3 network simulator. In simulation, the data transfer tested between the remote host and the PG-W. The simulation results show that Codel has a higher efficiency than RED, Pie, and PFIFO [13].

When the literature is examined, it is seen that many studies have been done on wired and wireless networks regarding queuing management algorithms. However, the queue management algorithms in LTE networks have been done in the last two years and these studies usually focus on mathematical analysis and versions of the RED algorithm. Unlike previous studies, in this study, we focused on performance analysis of each algorithm in LTE networks using NS-3 network simulator. In addition, the simulation study was done by keeping each algorithm's own parameters and according to different User Equipment Number.

\section{BACKGROUND}

\subsection{LTE Networks}

LTE offers a radio access network and an air interface and that provides low latency, high efficiency, improved system capacity and coverage [13]. LTE system was developed using the flat architectural structure of System Architecture Evolution (SAE). Cellular LTE network enables high-speed data transfer and signal transmission when handover occurs. As shown in Fig.1, LTE network architecture consists of two basic units, the core network and the evolved packet core (EPC) [14]. The IP Multimedia Core Network Subsystem (IMS) accesses the network with its EPC. Serving Gateway (SGW), Packet Data Gateway (PGW) and Mobility Management Entity (MME) are key units of the EPC. MME is responsible for the UE's user mobility and call processing after connection is established [15]. Serving Gateway (SGW) is tasked with routing and forwarding user data packets between LTE nodes and managing the transition between different cellular networks. PDN Gateway (PGW) connects the cellular network with the rest of the Internet. It also provides packet filtering, packet scanning and pricing support for each user. Home Subscriber Server (HSS) is a kind of database that also serves in the EPC. LTE network uses two types of nodes: UE (end-user) and eNodeB (base station). eNodeB nodes, connected to each other by $\mathrm{X} 2$ interface [16].

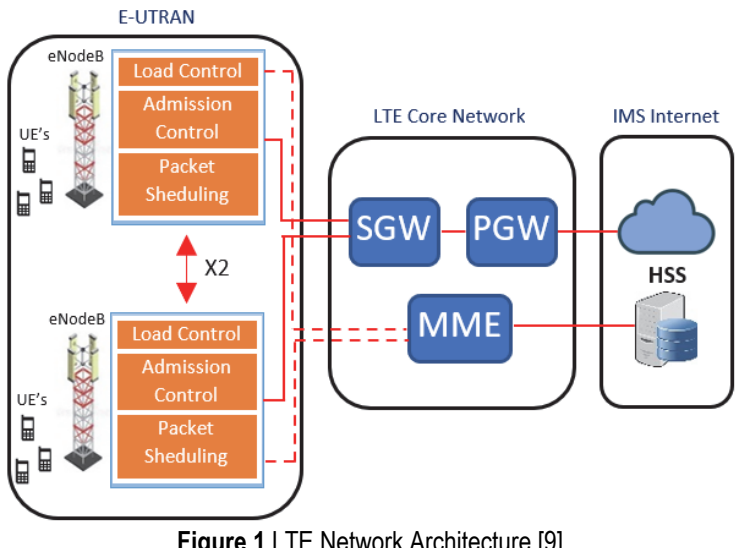

Figure 1 LTE Network Architecture [9]

\subsection{Queue Management Algorithms}

Many queue management algorithms have been developed to relieve intense traffic computer networks and to reduce potential bottlenecks. The most used queue management algorithms in-network with real-time applications and voice transmission are traditional and low latency ones. Active queue management algorithms try detecting the bottleneck by looking at the queue length when performing operations such as dropping packets on the queue.

\subsubsection{Droptail}

In the Droptail algorithm, which is based on queue management techniques, packets arriving up to the queue maximum length are included in the queue. Packets are rejected when the queue reaches maximum length. When the packet is sent from the queue and the queue is opened, new packets are added back to the queue. There is no effective management in this algorithm. The traffic from different sources is not differentiated. All decision making is based on whether the length of the queue is full or not [17]. Droptail queue management algorithm cannot work effectively in high-density traffic. Real-time traffic has high packet transmission. When many packets arrive in succession from the same stream and the queue is full, many packets from the same stream can be lost.

\subsubsection{RED}

The RED algorithm is the first active queue management developed in 1993. The RED algorithm is one 
of the widely used active queue management control mechanisms that work through routers. This algorithm is a queue size-based algorithm that processes according to the minimum and maximum threshold values [18]. For each packet arriving in the RED algorithm, the average queue size is calculated, and congestion is detected at the beginning. If the calculated average queue size is above the specified maximum threshold value, the packet is dropped immediately. If the calculated average queue size is between the maximum and minimum threshold values, the probability of dropping the package is calculated. Depending on the probability of dropping the package, the package is added to the queue or the package is dropped from the queue [19]. Eq. (1) shows the calculation of the average queue size.

$$
q_{\mathrm{avg}}=\left(1-w_{\mathrm{p}}\right) q_{\mathrm{avg}}+w_{\mathrm{p}} q
$$

The $q$ value in this formula is the instant queue size observed in the router. The $q_{\text {avg }}$ value is the old average queue size applied by the low-pass filter weight. When the $w_{\mathrm{p}}$ value increases, it affects the current queue size on the $q_{\text {avg }}$ of the queue. The RED low-pass filter is used to calculate the average queue length. In this case, sudden increases in queue length do not affect average queue length at significant levels. The $w_{\mathrm{p}}$ coefficient is the time constant of the low-pass filter. If the $w_{\mathrm{p}}$ coefficient is large, the average process will not filter sudden congestion [20].

The average queue length is calculated with two threshold values, minimum threshold $\left(\min _{\mathrm{th}}\right)$ and maximum threshold $\left(\max _{\mathrm{th}}\right)$. If the average queue length is lower than the maximum threshold, no packet can be marked. If the average queue length is greater than the maximum threshold, all incoming packets are marked. If the average queue length is between the minimum and maximum packet, it is calculated and marked [9-18]. The calculation of the probability of $p_{\mathrm{a}}$ is shown in Eq. (2).

$$
p_{\mathrm{a}}=p_{\max } \frac{\left(q_{\mathrm{avg}}-\min _{\mathrm{th}}\right)}{\left(\max _{\mathrm{th}}-\min _{\mathrm{th}}\right)}
$$

Here, $p_{\mathrm{a}}$ is a function of average queue length. The probability of marking a packet from a link is proportional to the bandwidth utilization rate of the $p_{\text {a }}$ link.

The average queue size is calculated for each packet in the RED algorithm. If the average queue size is less than the predetermined minimum threshold, the incoming packet is added to the queue. If the average queue size is between the minimum and maximum threshold values, the probability of packet dropping is calculated, and if the probability is high, the incoming packet is dropped. If it is not probable, it is added to the queue. If the average queue size is greater than the maximum threshold, the packet is dropped [21].

\subsubsection{CoDel}

The CoDel algorithm is a new Active Queue Management (AQM) mechanism proposed by Nichols and Jacobson that aims to efficiency control of the bufferbloat problem. The bufferbloat causes the package drop. Also, bufferbloat causes packet drops to accumulate in the queue even if it has a high buffer size.

Unlike other RED-Based AQM mechanisms, CoDel is independent of network parameters such as queue size, queue size average, queue threshold, drop rate, delay. CoDel predicts a bottleneck by using the packet delay time that caused the actual delay in the router queue. CoDel detects bottlenecks if the packet duration exceeds the target value for a specified time interval. After congestion is detected, the signals to drop the packet are given to control the queue growth [22].

\subsection{4 pFIFO}

This queue management algorithm is based on the FIFO algorithm. Traffic is classified with different channels. Higher priority traffic is processed faster [23]. The pFIFO queue management algorithm is designed as a simple method to support differentiated service classes and is the basis for queuing scheduling algorithms. The transmission of low computational load and the traffic generated by real-time applications can be shown as an advantage of the pFIFO queue management algorithm. One of the biggest problems with pFIFO is that you have high priority traffic in excessive amounts. If the high priority traffic volume becomes excessive, the buffer area allocated for low priority traffic falls and overflow begins. This causes low-priority traffic packets to fall [24].

\section{SIMULATION FRAMEWORK}

One of the easiest and the most effective ways of experimentally observing networks is the simulation method [13]. With this method, network nodes, connections, and network traffic are designed to be like the real world, and simulations of different situations can be easily performed [25].

NS-3 is an open-source discrete-event network simulator targeted primarily for research and educational use. NS-3 is licensed under the GNU GPLv2 license and can be used for research and development [26]. NS-3 is not retrospectively compatible with NS-2. Network Simulator 3 , used in the study, is an open-source discrete-event network simulator that provides the infrastructure for network research. NS-3 supports common network protocols and provides simulation results for the wired and wireless network. NS-3 is made by using $\mathrm{C}++$ and Python languages. The code structures are implemented through a Doxygen named documentation program [27].

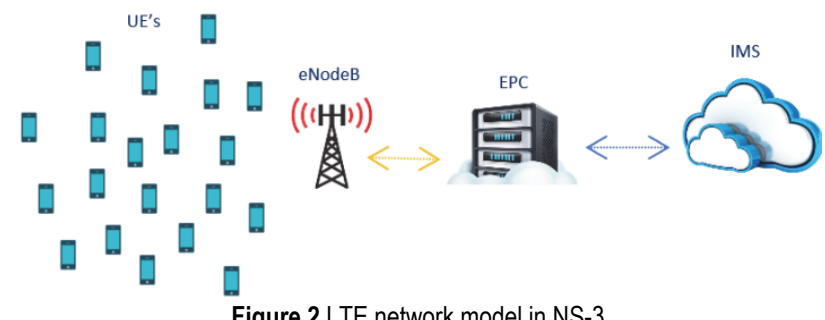

LENA (LTE-EPC Network Simulator) has been developed for the NS-3 LTE module. The LTE-EPC model has two main components, LTE and EPC. The EPC model 
provides tools for simulating end-to-end IP connectivity over the LTE model. A single SGW/PGW node is bound to more than one eNodeB and the data flow is through SGW and PGW [28, 29].

A sample LTE model is needed to test the queue management algorithms. In the LTE network model in Fig.2, there are 20 user equipments (UE), 1 eNodeB that these users are connected to, S-GW and P-GW nodes and internet node for connecting to IP Multimedia Subsystem (IMS) and services.

\subsection{Simulation Parameters}

The parameters in Tab. 1 are used for the LTE model. The data rate of the interface between the internet node and the S-GW/P-GW nodes is set to $100 \mathrm{Gbps}$. MTU (Maximum Transfer Unit) value for Ethernet networks are 1500 Bytes. This Ethernet indicates that a packet entering the network may have a maximum size of 1500 bytes. The delay between these nodes is 0.010 seconds. Wired link capacity is $100 \mathrm{Mbps}$. TCP traffic type is TCP NewReno. Users mobility type is RandomWalk2D. The interface between the S-GW/P-GW node and a the eNodeB node has a data rate of $100 \mathrm{Gbps}$ and a delay of $2 \mathrm{~ms}$ [28-30].

\begin{tabular}{|c|c|c|}
\hline & Parameters & Value \\
\hline \multirow[t]{11}{*}{ LTE } & $\begin{array}{l}\text { For internet - S-GW/P-GW } \\
\text { Node; }\end{array}$ & \\
\hline & $\begin{array}{l}\text { MTU (Maximum Transmission } \\
\text { Unit) }\end{array}$ & 1500 Bytes \\
\hline & Data Rate & $100 \mathrm{Gbps}$ \\
\hline & Delay & $0.010 \mathrm{~s}$ \\
\hline & $\begin{array}{l}\text { For S-GW/P-GW - eNodeB } \\
\text { Node; }\end{array}$ & \\
\hline & Wired Link Capacity & $100 \mathrm{Mbps}$ \\
\hline & Data Rate & $100 \mathrm{Gbps}$ \\
\hline & Delay & $2 \mathrm{~ms}$ \\
\hline & User Equipment Number & $20,40,60,80$ \\
\hline & TCP Traffic Type & TCP NewReno \\
\hline & Mobility & RandomWalk2D \\
\hline \multirow[t]{2}{*}{ Droptail } & Mode (Bytes, Packets) & Packets \\
\hline & MaxPackets & 50 \\
\hline \multirow[t]{4}{*}{ CoDel } & Mode (Bytes, Packets) & Packets \\
\hline & MaxPackets & 50 \\
\hline & Interval & $100 \mathrm{~ms}$ \\
\hline & Target & $5 \mathrm{~ms}$ \\
\hline \multirow[t]{7}{*}{ RED } & Mode (Bytes, Packets) & Packets \\
\hline & MeanPktSize & 50 \\
\hline & IdlePktSize & $1500 * 1000$ bytes \\
\hline & MinTh, MaxTh & 20,50 \\
\hline & QueueLimit & 50 \\
\hline & Queue weight & $0,002 \mathrm{~s}$ \\
\hline & LinkDelay & $20 \mathrm{~ms}$ \\
\hline pFIFO & Limit & 50 \\
\hline
\end{tabular}

The mode parameter used during the analysis of the Droptail queue management algorithm is chosen to process the queue as packets or bytes. MaxPackets parameter is the size of the queue [26-31].

The Mode parameter used during the analysis of the CoDel queue management algorithm is chosen to process the queue as packets or bytes. MaxPackets is the maximum number of packets that the queue can hold. The interval parameter sets the waiting time of the packets in the queue. Target parameters are used for the targets queue delay. The estimated target value is $5 \mathrm{~ms}$ [32].
The mode parameter used during the analysis of the RED queue management algorithm is chosen to process the queue as packets or bytes. MeanPktSize is the average queue size. IdlePktSize is the average packet size used during idle periods. The MinTh and MaxTh parameters are the maximum and minimum threshold values. The LinkDelay parameter is the connection delay value used for the RED algorithm [33].

The Limit parameter used during the analysis of the pFIFO queue management algorithm gives the queue size. A low value of this performance parameter indicates a low packet low rate. For this, the number of packets dropped on the queue must be minimized and the number of packets that successfully drop the queue must be as large as possible.

\subsection{Simulation Study}

We simulated Droptail, RED, CoDel and pFIFO algorithms with 20, 40, 60 and 80 users in LTE environment. End-to-end average throughput values for all algorithms are shown in Tab. 2. Droptail algorithm is the slowest running algorithm in terms of average end-to-end throughput value.

\begin{tabular}{|c|c|c|c|c|}
\hline \multirow{2}{*}{ AQM } & \multicolumn{4}{|c|}{ Average Throughput / Kbps } \\
\hline & $20 \mathrm{UE}$ & $40 \mathrm{UE}$ & $60 \mathrm{UE}$ & $80 \mathrm{UE}$ \\
\hline Droptail & 2520.99 & 1817.67 & 1217.57 & 513.67 \\
\hline RED & 2936.19 & 2138.11 & 1481.49 & 1014.87 \\
\hline CoDel & 2612.21 & 1934.13 & 1278.43 & 899.11 \\
\hline pFIFO & 2593.18 & 1987.71 & 1236.61 & 756.21 \\
\hline
\end{tabular}

The graph of average throughput values is shown in Fig. 3. Among all algorithms, the RED algorithm gave the best result according to the end-to-end efficiency value. The RED algorithm produces the best result in average throughput value with early detection of package drops and following average queue size. The closest result of the RED algorithm is the CoDel algorithm. Droptail algorithm, which drops the incoming packets after the queue is full, gave the worst result among all algorithms. In addition, Droptail gave worse results when the number of users increased. This shows that Droptail is the most vulnerable algorithm for high traffic.

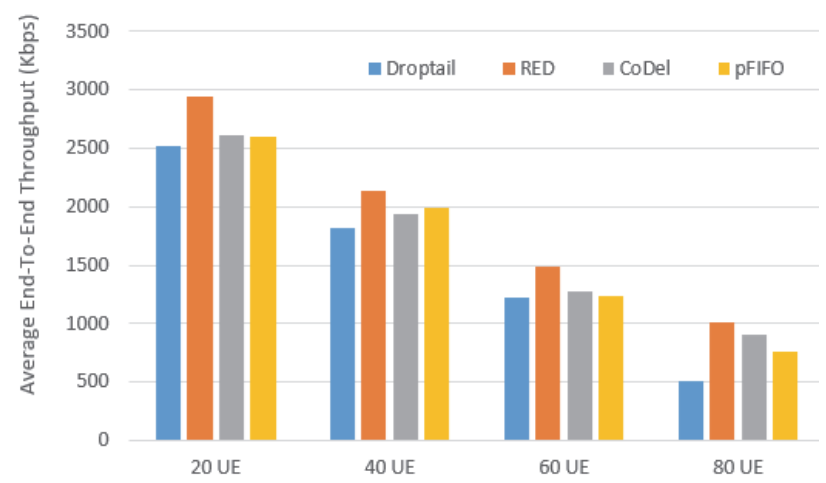

Figure 3 Average end-to-end throughput values for different AQM

According to Tab. 3, as the number of users increases the packet drops decrease. The number of packets dropped by the Droptail, Codel and pFIFO algorithms increases with the number of users, and therefore with the number of 
incoming packets. For this reason, these three algorithms do the packet dropping depending on the queue size. The RED algorithm takes the queue average for each incoming packet dropping according to the determined maximum and minimum thresholds. Likewise, as the queue size decreases, the number of packets dropped by the queue management algorithms increases.

Table 3 Arrival and dropping packets according to types of algorithms

\begin{tabular}{|c|c|c|c|c|c|c|c|c|}
\hline $\begin{array}{c}\text { Number of } \\
\text { UF }\end{array}$ & \multicolumn{2}{|c|}{$20 \mathrm{UE}$} & \multicolumn{2}{|c|}{$40 \mathrm{UE}$} & \multicolumn{2}{|c|}{$60 \mathrm{UE}$} & \multicolumn{2}{|c|}{$80 \mathrm{UE}$} \\
\hline Packets & 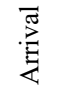 & 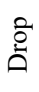 & 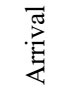 & $\begin{array}{l}\text { مे } \\
\text { مे }\end{array}$ & 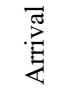 & $\begin{array}{l}\text { Oे } \\
\text { مً }\end{array}$ & 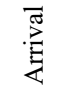 & 官 \\
\hline Droptail & 826 & 56 & 1650 & 210 & 2406 & 480 & 3260 & 560 \\
\hline RED & 837 & 20 & 1530 & 45 & 2389 & 110 & 3215 & 206 \\
\hline CoDel & 845 & 35 & 1635 & 150 & 2412 & 350 & 3219 & 350 \\
\hline pFIFO & 832 & 53 & 1585 & 205 & 2395 & 465 & 3189 & 535 \\
\hline
\end{tabular}

Fig. 4 shows the packet loss rate by percentage (\%) according to the algorithms and users numbers. The packets loss rate was found by dividing the number of packets dropped by the number of incoming packets. Although the performance varies according to all user number groups, the RED algorithm gives the best performance for all groups. The RED algorithm is followed by CoDel, pFIFO and Droptail algorithms in this order.

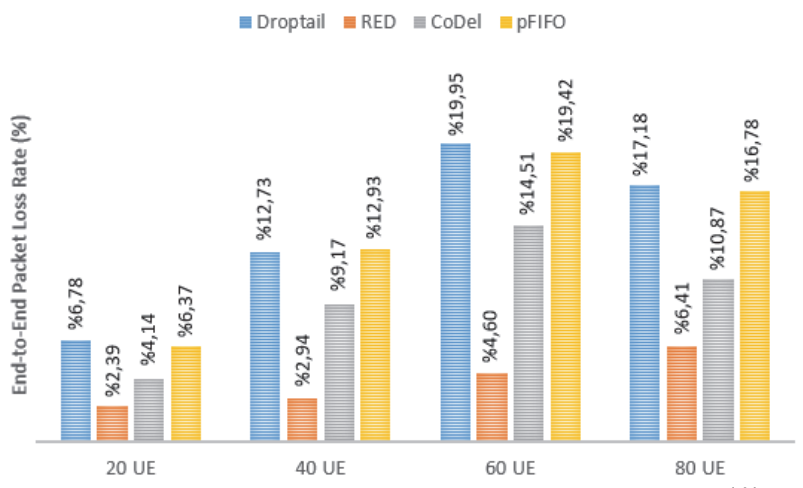

Figure 4 Packet loss rate based on user counts and algorithm types / \%

Less end-to-end delay in the LTE network allows users to access data in a short time. According to Tab. 4, the average end-to-end delay increases as the number of users increses. In the Droptail, Codel and pFIFO algorithms, the end-to-end delay occurs late due to the long response time in the packet drop mechanisms. The latency is less because the RED algorithm drops packets by calculating the average queue size. In addition, the RED algorithm causes fewer delays in the network because it drops fewer packets than other algorithms.

\begin{tabular}{|c|c|c|c|c|}
\hline \multirow{2}{*}{ AQM } & \multicolumn{5}{|c|}{ Average Delay / ms } \\
\cline { 2 - 5 } & $\mathbf{2 0}$ UE & $\mathbf{4 0}$ UE & $\mathbf{6 0 ~ U E}$ & $\mathbf{8 0}$ UE \\
\hline Droptail & 110 & 190 & 260 & 305 \\
\hline RED & 50 & 75 & 90 & 110 \\
\hline CoDel & 90 & 120 & 160 & 205 \\
\hline pFIFO & 103 & 175 & 249 & 298 \\
\hline
\end{tabular}

Fig. 5 shows average end-to-end delay times according to the user's numbers. The end-to-end delay depends on the number of routers on the path between the source and destination. Processes such as modulation, coding, packet creation time and queue management in the router are significantly increasing the end-to-end delay. Since the RED algorithm has the lowest packet loss rate, the end-toend delay value is also low.

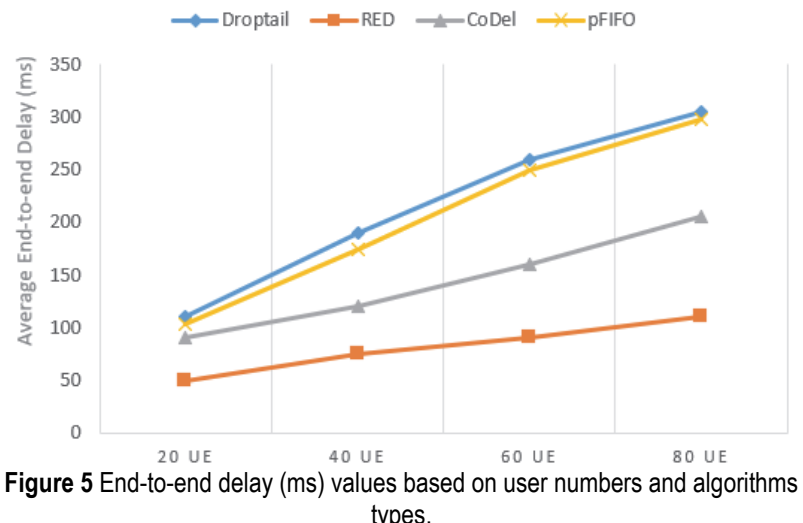

Tab. 5 shows the variation of the Packet delivery fraction (PDF) rate by the number of users. PDF, calculated by the ratio of the total sent packets to total received packets, is an important ratio showing the performance of the network.

Table 5 Average PDF rate / \%

\begin{tabular}{|c|c|c|c|c|}
\hline \multirow{2}{*}{ AQM } & \multicolumn{4}{|c|}{ Average PDF / \% } \\
\cline { 2 - 5 } & $\mathbf{2 0 ~ U E}$ & $\mathbf{4 0} \mathbf{~ U E}$ & $\mathbf{6 0 ~ U E}$ & $\mathbf{8 0}$ UE \\
\hline Droptail & 93.22 & 87.27 & 80.05 & 82.82 \\
\hline RED & 97.61 & 97.06 & 95.40 & 93.59 \\
\hline CoDel & 95.86 & 90.83 & 85.49 & 89.13 \\
\hline pFIFO & 93.63 & 87.07 & 80.58 & 83.22 \\
\hline
\end{tabular}

According to Fig. 6, RED is the algorithm with the largest number of packets in the eNodeB buffer. RED processes the packets according to the average queue length, which drops them from the queue. For this reason, it is the algorithm with the highest rate of packets sent and received. It is better than other algorithms in PDF value. Droptail is the algorithm with the lowest PDF rate because when the queue is full, it immediately drops packets that accumulate in the queue.

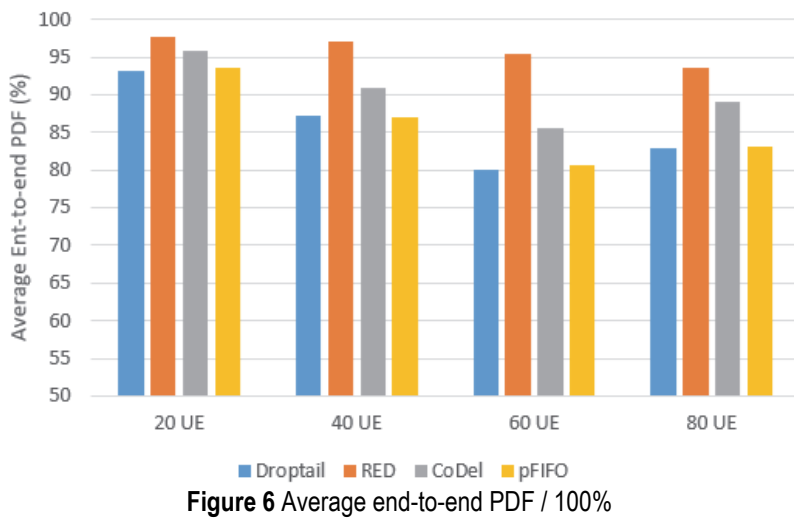

Packet congestion control mechanisms for the LTE network all transmission protocols or peer-to-peer 
implementation interact well with transmission protocols. Fairness index requires that a protocol takes on no larger share of the network than comparable flows. Tab. 6 shows the spread of fairness index values according to the number of users of Droptail, RED, CoDel and pFIFO algorithms.

Table 6 Fairness index for algorithms

\begin{tabular}{|c|c|c|c|c|}
\hline \multirow{2}{*}{ AQM } & \multicolumn{4}{|c|}{ Fairness Index } \\
\cline { 2 - 5 } & $\mathbf{2 0}$ UE & $\mathbf{4 0}$ UE & 60 UE & 80 UE \\
\hline Droptail & 0.85 & 0.77 & 0.71 & 0.59 \\
\hline RED & 0.98 & 0.95 & 0.91 & 0.86 \\
\hline CoDel & 0.91 & 0.89 & 0.83 & 0.70 \\
\hline pFIFO & 0.87 & 0.81 & 0.75 & 0.68 \\
\hline
\end{tabular}

According to Fig. 7, the fairness index value is the highest of the RED algorithm. RED allows maximum resource allocation for the network by minimizing queue waiting time. Sharing resources on the LTE network directly affects service time and QoS quality. RED maximizes the QoS value on the network by dropping fewer packets. Droptail has the worst fairness index value due to the uncontrolled package drop mechanism.

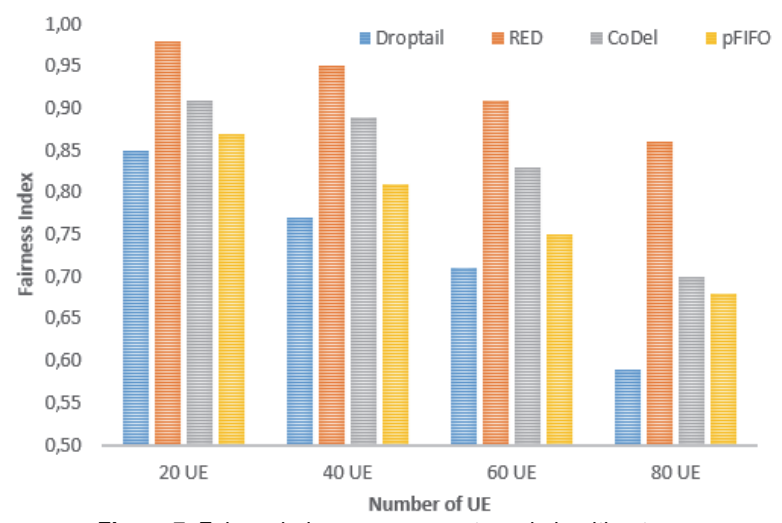

Figure 7 Fairnex index on user counts and algorithm types

We simulated Droptail, RED, CoDel and pFIFO algorithms for 80 users based on different Transmission Time Interval (TTI) values. Tab. 7 shows the average throughput results for TTI times for 10, 20, 30 and $40 \mathrm{~ms}$. Using different TTI times, we measured the effect of transmission time on the traffic between packets in the LTE environment. The accumulation of packets in the buffer is directly proportional to the TTI duration.

Table 7 Performance of the system for the target time interval

\begin{tabular}{|c|c|c|c|c|}
\hline \multirow{2}{*}{$\begin{array}{c}\text { Target } \\
\text { Time } \\
\text { Interval / } \\
\text { ms }\end{array}$} & \multicolumn{4}{|c|}{ Average Throughput for 80 UE / Kbps } \\
\cline { 2 - 5 } & Droptail & RED & CoDel & pFIFO \\
\hline 10 & 513.67 & 1014.87 & 899.11 & 756.21 \\
\hline 20 & 498.11 & 1001.67 & 887.78 & 741.37 \\
\hline 30 & 461.89 & 993.89 & 867.29 & 723.14 \\
\hline 40 & 430.19 & 989.13 & 861.13 & 701.21 \\
\hline
\end{tabular}

As the TTI time increases, the number of packets waiting in the eNodeB buffer decreases. Less packet accumulation in the buffer reduces packet drops. In this case, the response time of the network is delayed. All algorithms produced close results on average. Only the buffer accumulation times have increased. RED produced the best result because it used the average queue size. The CoDel, pFIFO and Droptail algorithms, respectively, produce the best results (Fig. 8).

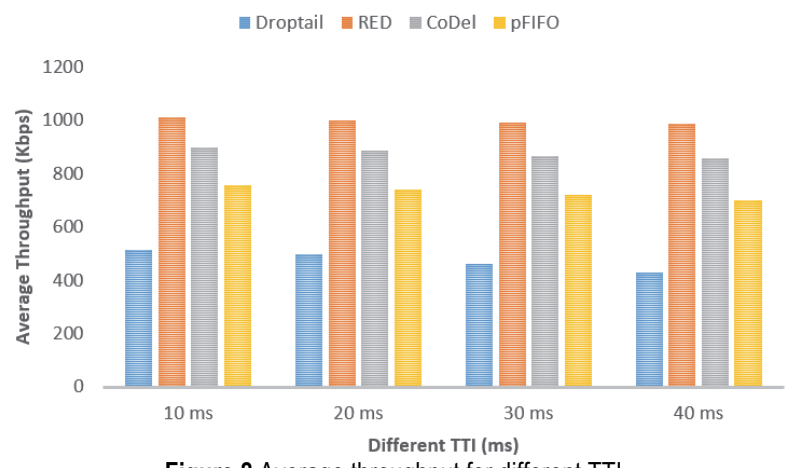

Figure 8 Average throughput for different TTI

\subsection{Simulation Results}

In the simulation studies, the RED queue management algorithm produces the best results in average throughput, delay, packet loss rate, PDF and fairness index rates. CoDel queue management algorithm performs packet dropping with reference to the queue waiting time of the packet. Even if there is a possibility of overflowing the queue and dropping all incoming packets, the packet is not dropped unless the packet waiting time in the queue has exceeded the release time. The Droptail and pFIFO algorithms are traditional queue management algorithms and the reduction of the package depends on whether or not the queue is full. These are the reasons why the three algorithms show lower performance than the RED queue management algorithm. The RED queue management algorithm controls the length of the queue and decides whether to reduce the packet depending on the specified minimum and maximum threshold values. The advantages of routers using the RED algorithm over side stream routers have been accepted by many researchers. However, when the congestion is too heavy, the network device cannot control the average queue length and the average queue length can exceed the maximum threshold value. In this case, all incoming packets are dropped without considering any possibility. When the congestion suddenly concentrates, the actual queue length will increase and pass the queue boundary. However, as the average queue length is lower than the minimum threshold value, no packets will be dropped randomly.

\section{CONCLUSION}

The simulation results show that the most successful algorithm is the RED algorithm which yields the lowest packet loss rate according to the packet traffic. The RED algorithm is followed by CoDel, pFIFO and Droptail algorithms. This is because the RED algorithm calculates the average queue value for each incoming packet and decides whether the packet should be dropped according to the threshold values. Other algorithms look at the queue length to decide if the package can be dropped. In the places where resources are limited, these methods are not effective. The purpose of an appropriate active queue 
management configuration is to keep the average queue size between the minimum threshold, thereby avoiding compulsory packet drops. It is not appropriate that the RED algorithm is linearly related to the probability of packet drop and the average queue size. The link bandwidth is found not to be fully used with a small average delay in the low load scenario. Therefore, the possibility of a smaller packet dropping should be used to improve connection utilization. In a high load scenario, the bandwidth is fully used with a large average delay. For this reason, a larger packet drop probability should be used to reduce the average delay. Moreover, the computation of minimum and maximum threshold values, based on the traffic load, can increase the performance of the RED algorithm in LTE cellular system in low and heavy traffic loads.

\section{REFERENCES}

[1] Santos, R. A., Block, A. E., \& Licea, V. R. (2014) Broadband wireless access networks for 4G: Theory, application, and experimentation. Hershey: Information Science Reference (an imprint of IGI Global). https://doi.org/10.4018/978-1-4666-4888-3

[2] Ghulam Sarwar, G., Ullah, F., Lee, H.-W., Ryu, W., \& Lee, S. (2017). Quality of service and mobility aware in-vehicle telescreen service architecture. Tehnicki Vjesnik - Technical Gazette, 24(1). https://doi.org/10.17559/TV-20150819110655

[3] Kasapovic, S., Mujkic, S., \& Mujacic, S. (2016). Enhanced MIMO Influence on LTE-Advanced Network Performances.vvvElektronika Ir Elektrotechnika, 22(1). https://doi.org/10.5755/j01.eie.22.1.7698

[4] Rehman, E., Sher, M., Abass Naqvi, S. H., Khan, K. B., \& Ullah, K. (2019). Secure Cluster-Head Selection Algorithm Using Pattern for Wireless Mobile Sensor Networks. Tehnicki Vjesnik - Technical Gazette, 26(2). https://doi.org/10.17559/TV-20170319133811

[5] Reddy, T. B. \& Ahammed, A. (2008). Performance Comparison of Active Queue Management Techniques. Journal of Computer Science, 4(12), 1020-1023. https://doi.org/10.3844/jcssp.2008.1020.1023

[6] Ceco, A., Nosovic, N., \& Bradic, K. (2012). Performance comparison of active queue management algorithms. 2012 20th Telecommunications Forum (TELFOR). https://doi.org/10.1109/TELFOR.2012.6419188

[7] Priority Queuing and Scheduling Algorithms Analysis Using Opnet Modeler. (2012). International Conference on Computer and Automation Engineering, 4th (ICCAE 2012), 365-370. https://doi.org/10.1115/1.859940.paper57

[8] Albayrak, Z. \&.Torun, C. (2016). Recent LTE Simulation Tools. 2nd International Conference on Engineering and Natural Sciences (ICENS 2016).

[9] Paul, A., Kawakami, H., Tachibana, A., \& Hasegawa, T. (2017). Effect of AQM-Based RLC Buffer Management on the eNB Scheduling Algorithm in LTE Network. Technologies, 5(3), 59. https://doi.org/10.3390/technologies5030059

[10] Malathi, A. (2016). A Study on Performance Modelling and Analysis of Network on Chip under M-Port N-Tree Bursty Traffic. International Journal of Engineering and Computer Science. https://doi.org/10.18535/ijecs/v5i10.38

[11] Zarrini, M. \& Ghasemi, A. (2018). Loss and delay analysis of non-Poisson M2M traffic over LTE networks. Transactions on Emerging Telecommunications Technologies, 29(2). https://doi.org/10.1002/ett.3273

[12] Adesh, N. D. \& Renuka, A. (2019). Avoiding queue overflow and reducing queuing delay at eNodeB in LTE networks using congestion feedback mechanism. Computer Communications, 146, 131-143. https://doi.org/10.1016/j.comcom.2019.07.015

[13] Çakmak, M. \& Albayrak, Z. LTE Ağlarda Remote-Host ile PG-W Arasındaki Kuyruk Yönetim Algoritmalarının Performans Analizi. Akademik Platform Mühendislik ve Fen Bilimleri Dergisi, 8(3), 456-463. https://doi.org/10.21541/apjes.662677

[14] Çakmak, M., \& Albayrak, Z. (2018). A Review: Mobile Communication Past, Present and Future. International Conference on Advanced Technologies, Computer Engineering and Science, 141-145.

[15] Sesia, S., Toufik, I., \& Baker, M. (2015). LTE - the UMTS long term evolution: From theory to practice. Hoboken: John Wiley \& Sons.

[16] Akyildiz, I. F., Gutierrez-Estevez, D. M., Balakrishnan, R., \& Chavarria-Reyes, E. (2014). LTE-Advanced and the evolution to Beyond 4G (B4G) systems. Physical Communication, 10, 31-60. https://doi.org/10.1016/j.phycom.2013.11.009

[17] Patil, G., Mcclean, S., \& Raina, G. (2011). Drop Tail And Red Queue Management With Small Buffers: stability And Hopf Bifurcation. ICTACT Journal on Communication Technology, 02(02), 339-344. https://doi.org/10.21917/ijct.2011.0047

[18] Floyd, S. \& Jacobson, V. (1993). Random early detection gateways for congestion avoidance. IEEE/ACM Transactions on Networking, 1(4), 397-413. https://doi.org/10.1109/90.251892

[19] Çakmak, M. \& Albayrak, Z. (2018). A Review: Active Queue Management Algorithms in Mobile Communication. 2018 International Conference on Advanced Technologies, Computer Engineering and Science (ICONCS), 180-184.

[20] Nichols, K. \& Jacobson, V. (2012). Controlling Queue Delay. Queue, 10(5), 20. https://doi.org/10.1145/2208917.2209336

[21] Al-Raddady, F. \& Woodward, M. (2007). A New Adaptive Congestion Control Mechanism for the Internet Based on RED. 21st International Conference on Advanced Information Networking and Applications Workshops (AINAW'07). https://doi.org/10.1109/AINAW.2007.31

[22] Raghuvanshi, D. M., Annappa, B., \& Tahiliani, M. P. (2013). On the Effectiveness of CoDel for Active Queue Management. 2013 Third International Conference on Advanced Computing and Communication Technologies (ACCT). https://doi.org/10.1109/ACCT.2013.27

[23] Yap, K. M. \& Marshall, A. (2010). Investigating Quality of Service Issues for Distributed Haptic Virtual Environments in IP Networks. 2010 Sixth International Conference on Intelligent Environments. https://doi.org/10.1109/IE.2010.50

[24] Mustafa, M. E. \& Talab, S. A. (2016). The Effect of Queuing Mechanisms First in First out (FIFO), Priority Queuing (PQ) and Weighted Fair Queuing (WFQ) on Network's Routers and Applications. Wireless Sensor Network, 08(05), 77-84. https://doi.org/10.4236/wsn.2016.85008

[25] Zhao, Y., Yu, H., \& Liang, G. (2015). NS3-based simulation system in heterogeneous wireless network. 11th International Conference on Wireless Communications, Networking and Mobile Computing (WiCOM 2015).

[26] Riley, G. F. \& Henderson, T. R. (2010). The ns-3 Network Simulator. Modeling and Tools for Network Simulation, 1534. https://doi.org/10.1007/978-3-642-12331-3_2

[27] Baldo, N., Requena-Esteso, M., Miozzo, M., \& Kwan, R. (2013). An open source model for the simulation of LTE handover scenarios and algorithms in ns-3. Proceedings of the 16th ACM International Conference on Modeling, Analysis \& Simulation of Wireless and Mobile Systems MSWiM '13. https://doi.org/10.1145/2507924.2507940

[28] Perez, A. (2015). LTE and LTE advanced: $4 G$ network radio interface. Hoboken, NJ: Wiley. https://doi.org/10.1002/9781119145462 
[29] Long Term Evolution (LTE) and LTE-Advanced. (2014). From GSM to LTE-Advanced, 235-326. https://doi.org/10.1002/9781118861943.ch4

[30] Andreozzi, M. M. (2011). LTE and LTE-Advanced evaluation through innovative simulation tool. 2011 IEEE International Symposium on a World of Wireless, Mobile and Multimedia Networks.

https://doi.org/10.1109/WoWMoM.2011.5986199

[31] Altman, E. \& Jiménez, T. (2012). NS Simulator for Beginners. Synthesis Lectures on Communication Networks, 5(1), 1-184. https://doi.org/10.2200/S00397ED1V01Y201112CNT010

[32] Sun, S., Jiang, W., Feng, G., Qin, S., \& Yuan, Y. (2019). Cooperative Caching with Content Popularity Prediction for Mobile Edge Caching. Tehnicki Vjesnik - Technical Gazette, 26(2). https://doi.org/10.17559/TV-20190303092125

[33] Tiwana, M. I., Nawaz, S. J., Ikram, A. A., \& Tiwana, M. I. (2014). Self-Organizing Networks: A Packet Scheduling Approach for Coverage/Capacity Optimization in 4G Networks Using Reinforcement Learning. Elektronika Ir Elektrotechnika, 20(9).

https://doi.org/10.5755/j01.eee.20.9.4786

\section{Contact information:}

\section{Muhammet ÇAKMAK}

Karabuk University Department of Computing Engineering,

Baliklar Kayasi Mevkii, 78050 Karabuk, Turkey

E-mail: muhammetcakmak@karabuk.edu.tr

\section{Zafer ALBAYRAK}

(Corresponding author)

Karabuk University Department of Computing Engineering,

Baliklar Kayasi Mevkii, 78050 Karabuk, Turkey

E-mail: zalbayrak@karabuk.edu.tr

\section{Cumhur TORUN}

Karabuk University Department of Computing Engineering,

Baliklar Kayasi Mevkii, 78050 Karabuk, Turkey

E-mail: cumhur.torun@kastamonumtal.k12.tr 